\title{
MANAGEMENT OF DIRECT INGUINAL HERNIA
}

\author{
WILLIAM A. DOWNES, M.D. \\ NEW YORK
}

The problem of inguinal hernia has resolved itself into the management of the direct variety. This statement is based on the fact that all surgeons agree that in indirect or oblique hernia the results following operation are excellent, provided it is performed properly and according to one of the approved methods. No such claim can be made for the operative results in direct hernia, nor can it be said that we have a standardized operation for this condition. Many surgeons recognize the futility of the usual technic for indirect hernia when applied to the direct type, and various modifications of recognized procedures have been devised in the effort to correct the inherent anatomic defects that give rise to this form of rupture. $U_{p}$ to the present time, however, none of these modifications have proved satisfactory in all cases, and I, for one, have reached the conclusion that a certain number of direct hernias cannot be cured by operation.

The difficulty lies in selecting the cases suitable for operative treatment. It is not an uncommon experience in what seemed to be a most favorable case to have the patient return with an early recurrence, and on the other hand, of having a most unfavorable case result in a permanent cure. As a rule, however, the patients in whom recurrence is almost sure to take place belong to a definite group. They are usually thin men with poorly developed muscles in both lower quadrants. Coughing or strain produces a general bulge extending from above the anterior superior spine over to the margin of the rectus, and down to the external ring, with a more or less marked hernial protrusion about the middle of the inguinal canal or at the ring. Palpation of the inguinal region may reveal a positive separation in the external oblique aponeurosis; or by invagination of the scrotum, one or more fingers may be inserted into the external ring and passed directly backward, revealing almost complete absence of the conjoined tendon. In other words, not only is the roof to the inguinal canal weak, but the floor is formed principally of transversalis fascia and peritoneum.

To the foregoing group may be added certain persons who have accumulated considerable fat over the lower abdomen. This fat obscures the condition of the underlying muscles and also interferes with proper palpation. The unfavorable outlook in this class of cases may not be recognized until after the patient comes to operation. The man with weak muscles and a small bulge at the lower part of the 
inguinal canal is not infrequently referred to the surgeon for operation by examiners for industrial corporations and civil service boards before being given employment. It would be far better in many instances for both the employer and the patient if a light truss was applied and a note made on the application form to the effect that the result following operation would probably prove unsatisfactory on account of poor muscles, that the slight hernia was in no way incapaci-

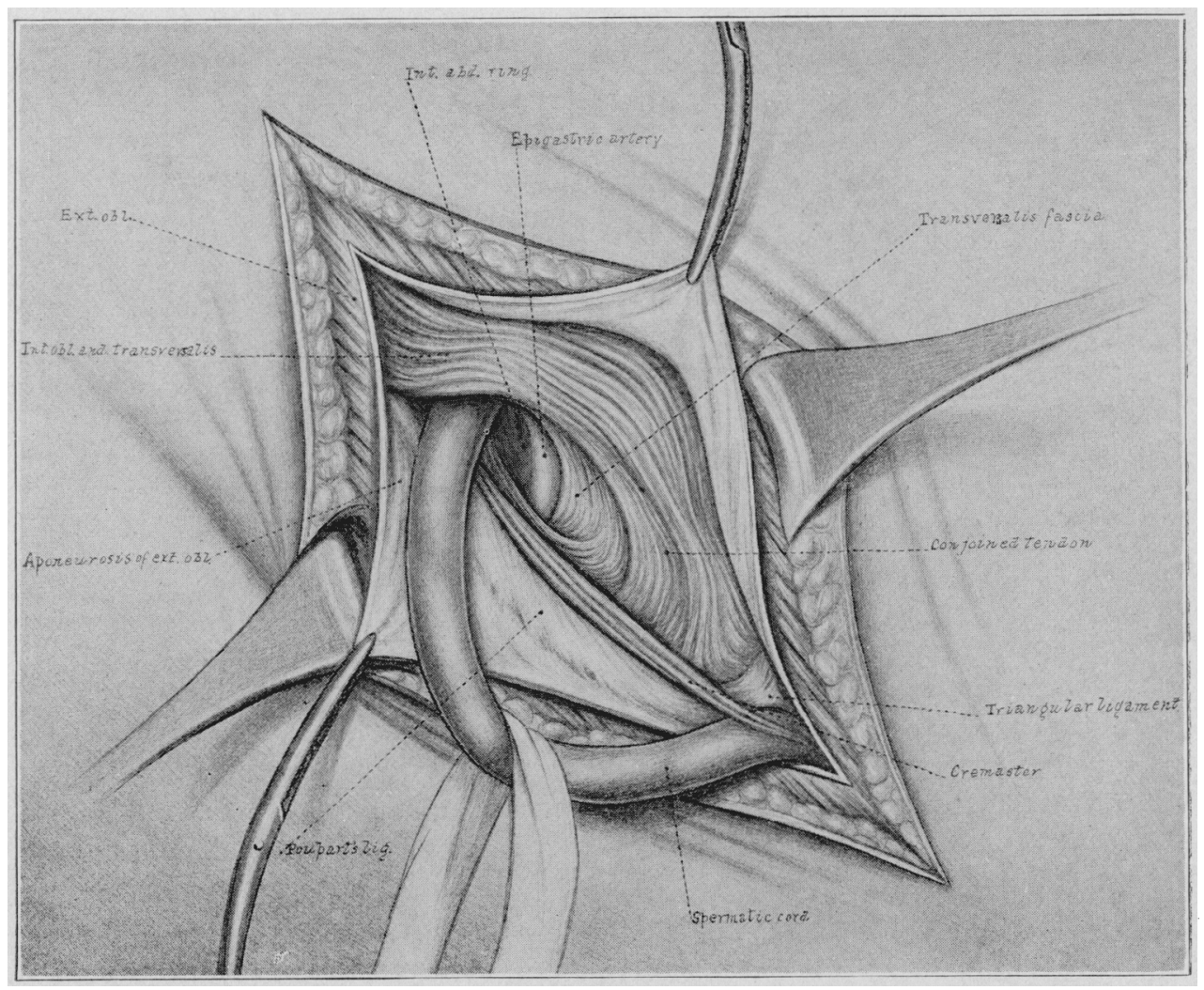

Fig. 1.-Anatomy of inguinal region.

tating, and that with proper support it would not be made worse by ordinary work. Let the employee accept the position with this under. standing and assume the responsibility. If, on the other hand, opera-. tion is performed with the chances of an early recurrence, the corporation or state becomes responsible.

No better evidence could be had of the value of the foregoing suggestion than the attitude taken by the officials responsible for the regulations governing the draft in our recent army. At first, any man 
was accepted by the army after he had undergone a so-called radical cure operation for hernia, without especial attention to the type of hernia, length of time following operation, or to the surgeon by whom it was performed. Later, all men between the ages of 21 and 31 with hernia were accepted by the army. If the time between examination and date of call permitted, these men could go where or to whom they chose for operation; if not, they were forced to be operated on immediately after reaching camp. Finally, in the last draft no man with

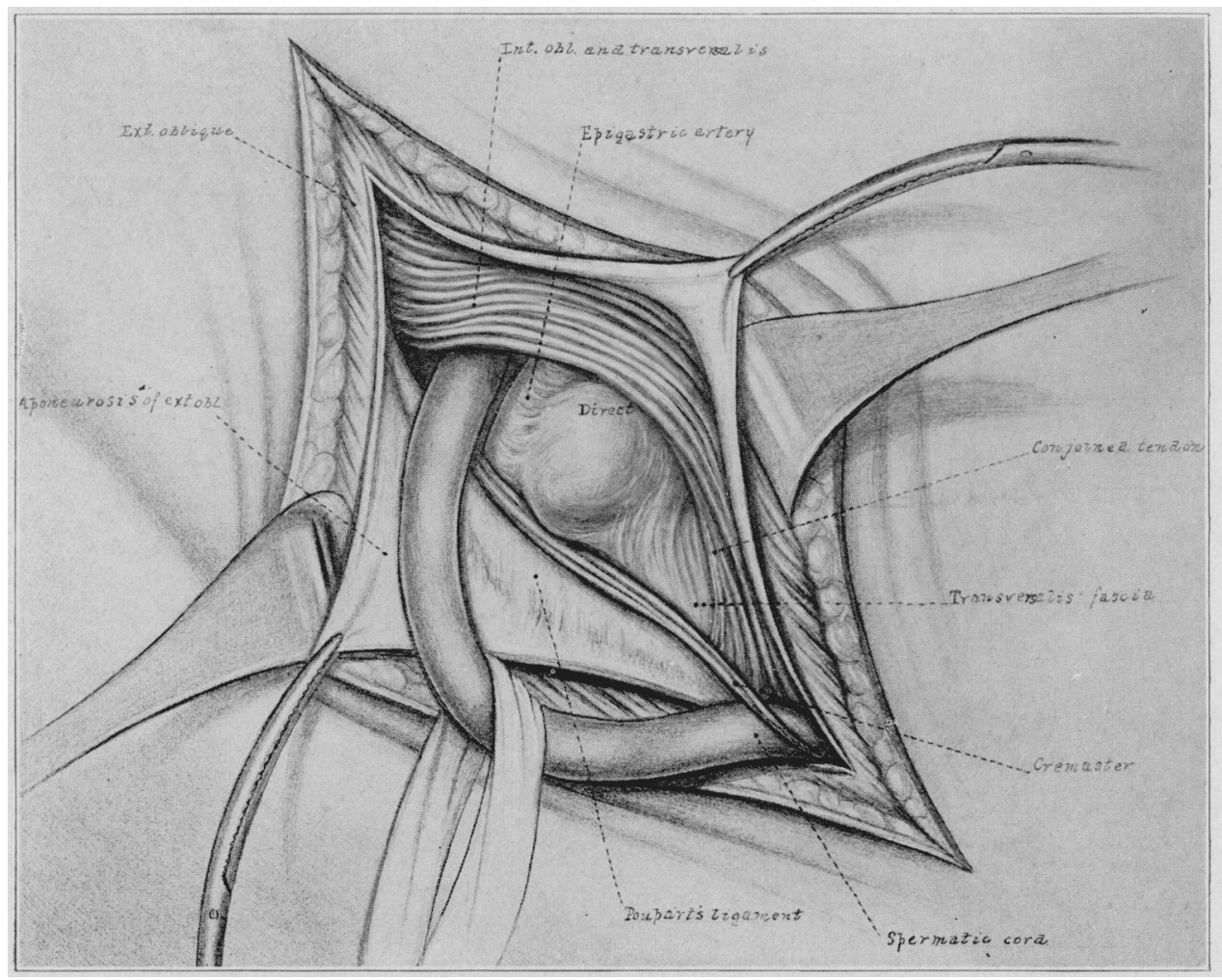

Fig. 2.-Direct inguinal hernia.

hernia was accepted for full military duty either before or after being operated on. From these changes in the regulations, it is quite evident that the results following herniotomy were not so satisfactory as had been anticipated. While some of the recurrences in soldiers may have been the result of poor technic, I venture to state that the great majority occurred in hernia of the direct variety, and that a good percentage of these were of the type which should never have been operated on. Aside from the disappointment to the surgeon in having recurrence take place, the patient himself deserves more consideration; 
for, after all, it is he who has to suffer the consequences. We often console ourselves with the thought that even if the hernia does return it will not be so bad or so hard to control by a truss as it was before operation. When the loss of time, the danger of going through an operative procedure, even though very slight, and the occasional risk of complications are taken into consideration, are we quite justified in operating except for good reason in this type of case when we know from experience that the result is likely to be unsatisfactory?

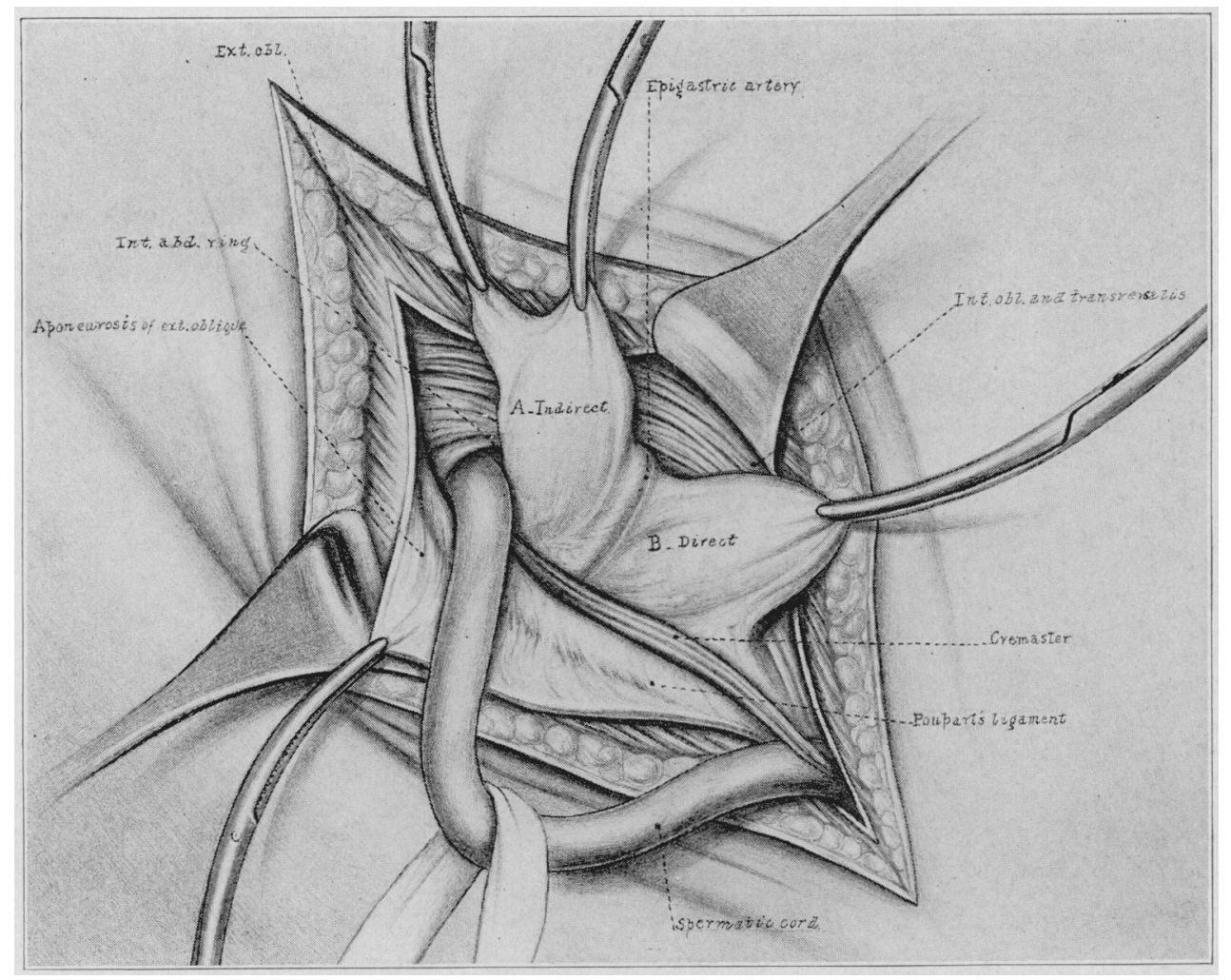

Fig. 3.-Combined direct and indirect hernia - "pantaloon sac."

\section{ANATOMIC CONSIDERATIONS}

A correct knowledge and clear understanding of the anatomy of the inguinal region (Fig. 1) is necessary in order to obtain satisfactory results from the operative treatment of hernia. Without this knowledge there is little satisfaction to the surgeon, and a grave injustice may be done the patient. Paradoxical as it may seem, the wonderful results obtained in recent years following operation in the indirect inguinal hernia group, which comprises more than 90 per cent. of all 
hernias, is largely responsible for the number of failures in the cases of direct hernia. The explanation is that many surgeons have come to look on the hernia operation as of almost minor importance, and pass these patients along to young assistants or house surgeons without proper supervision, with the result that if the anatomy or the arrangement of the sac is unusual, the operation is likely to prove a failure.

For the purposes of this paper, I shall speak of the inguinal region as that triangular part of the lower abdominal wall bounded below and to the outer side by Poupart's ligament, internally by the

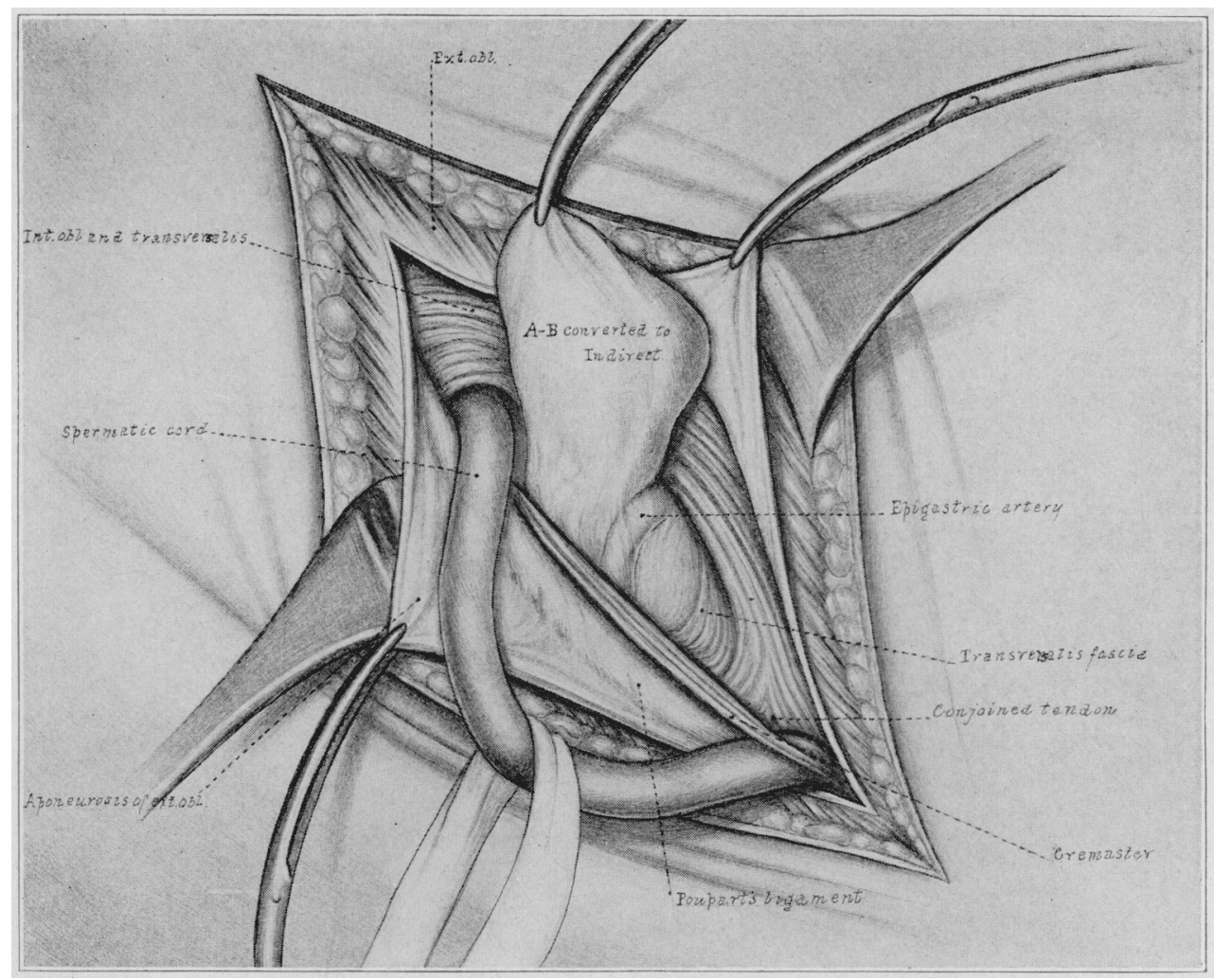

Fig. 4.-Combined sac converted into indirect hernia.

margin of the rectus, and above by a horizontal line extending from the anterior superior spine to the rectus muscle. The structures to be studied in the dissection of this area are the external oblique, internal oblique and transversalis muscles, including the cremaster muscle; the rectus muscle and its sheath, the conjoined tendon and transversalis fascia; also, the iliohypogastric and ilio-inguinal nerves, and the deep epigastric artery. 
The external oblique muscle, the fibers of which run down and in, may be referred to as the roof of the triangle. It is usually a firm aponeurotic structure extending as Poupart's ligament from the anterior superior spine to the pubic bone on the outer side, and to the linea alba on the inner. It presents at its lower inner portion an opening known as the external abdominal ring, which should normally admit the tip of the finger. The portions of the internal oblique and transversalis muscles encountered in the dissection arise from the

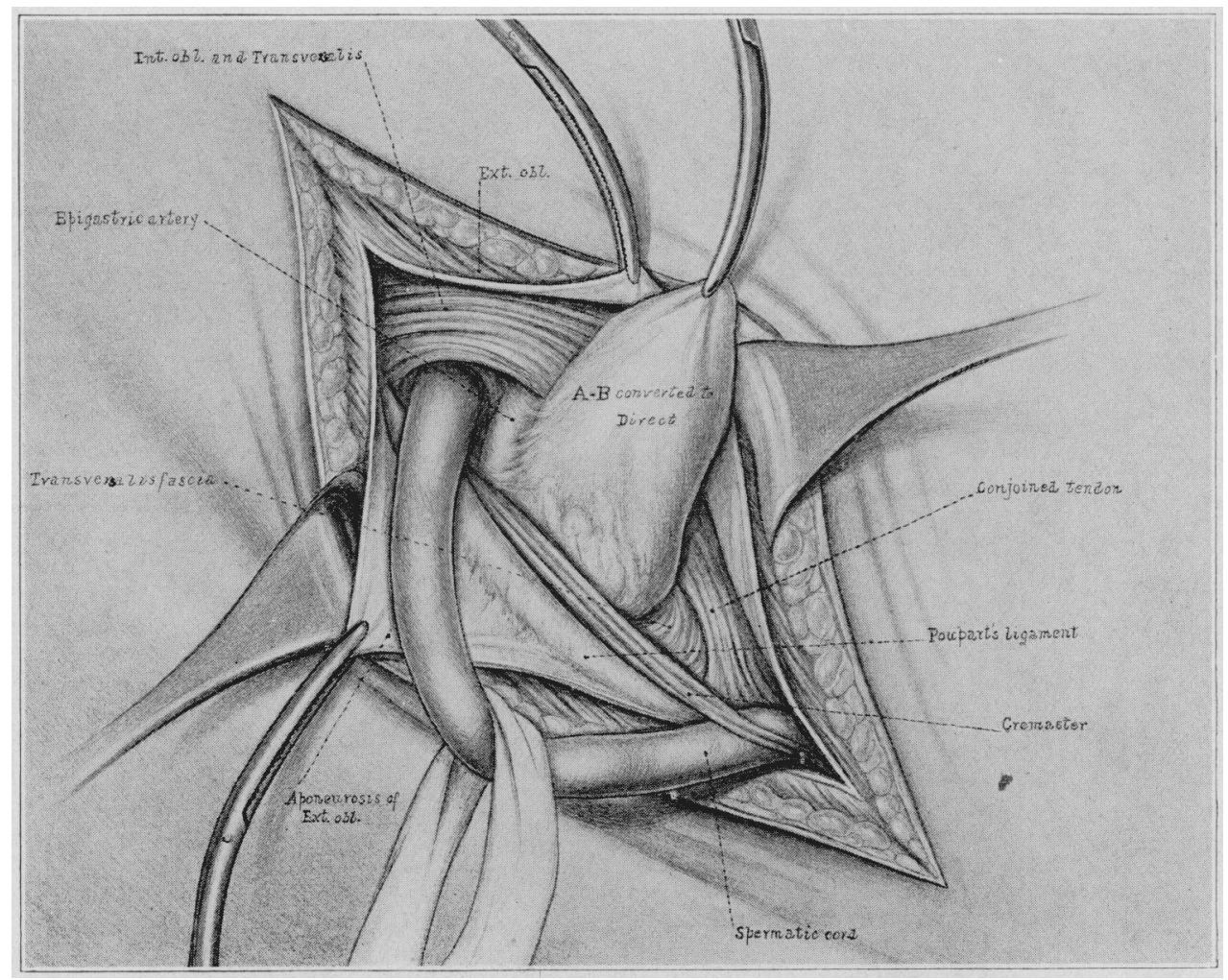

Fig. 5.-Combined sac converted into direct hernia.

outer half to the outer third of Poupart's ligament. The former usually appears as a well-developed muscle arching down and in, completely overlapping the latter. The two blend into a common aponeurosis, which becomes part of the anterior sheath of the rectus in its lower fourth and is continued down to be inserted into the pubic crest and pectineal line - the latter portion forming the conjoined tendon. This tendon, extending laterally from the margin of the rectus muscle, lies just behind the external ring and in front of the 
transversalis fascia. Its thickness and width vary in different persons. but when well developed it is of considerable value in the prevention of direct hernia. The cremaster, a thin layer of muscle fiber surrounding completely or in part the spermatic cord, has its origin at the lower margin of the internal oblique.

The transversalis fascia - a fairly thick fibro-aponeurotic structure - may be said to form the floor of this triangular area. The inner portion of this fascia is composed of more or less vertical fibers

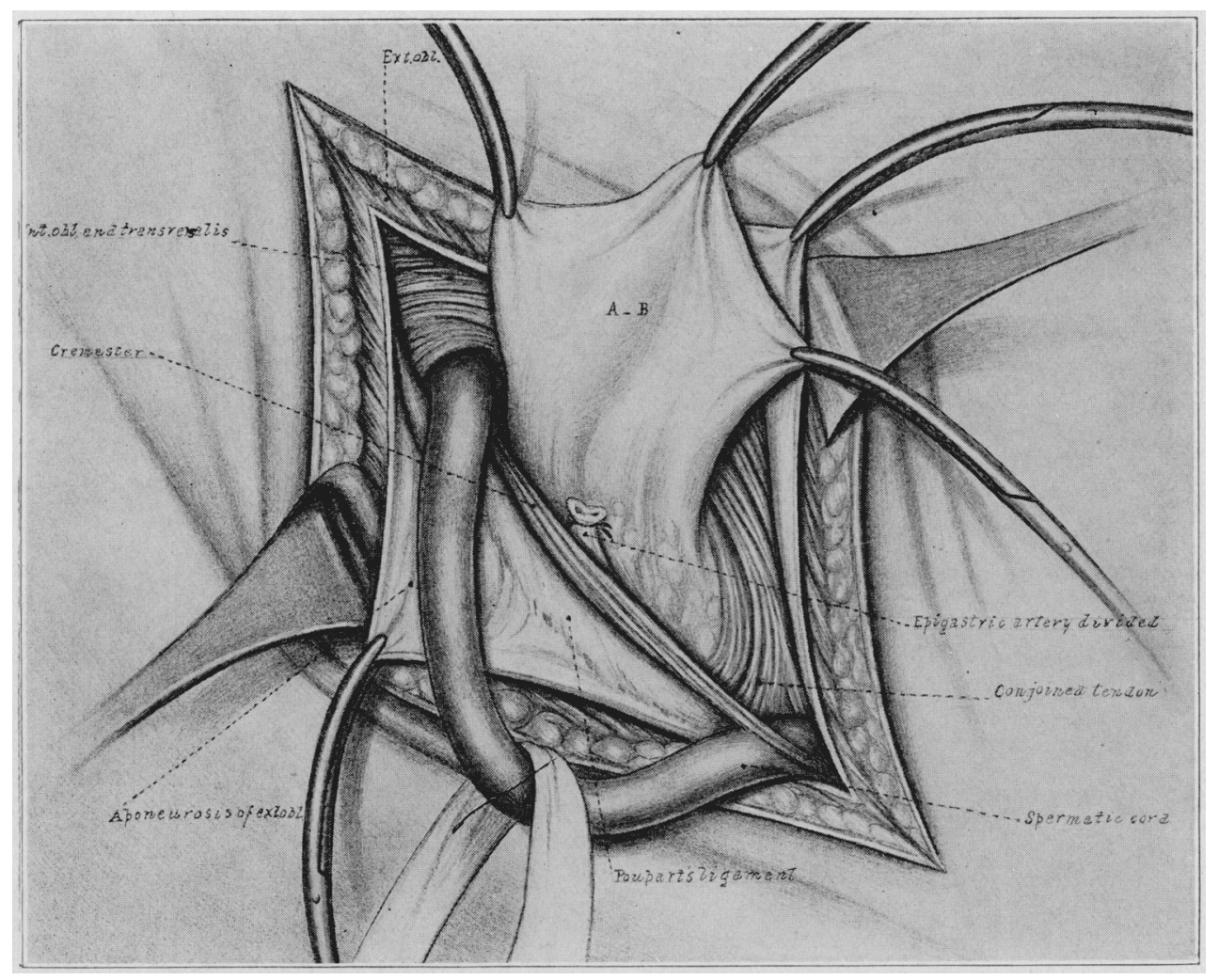

Fig. 6.-Combined sac converted into single sac by dividing epigastric artery.

of considerable strength; the outer is made up of arching fibers through which the cord passes, forming the internal ring. The middle portion, lying just below and internal to the deep epigastric artery, is the thinnest part of the fascia, and constitutes the weakest point in the floor. It is through this weak middle part that the great majority of direct hernias occur.

The deep epigastric artery - a branch of the external iliac - lies between the transversalis fascia and peritoneum, and runs on a line 
from the middle of Poupart's ligament to the umbilicus. This artery forms the lower boundary of the internal ring, and is the most important landmark in this region, as the variety of a given hernia is determined by the relation of the neck of its sac to this vessel. The iliohypogastric and ilio-inguinal nerves - the former running along the anterior surface of the internal oblique to the margin of the rectus muscle, and the latter through the inguinal canal to the external ring - are both sensory in this location, but should be preserved. The inguinal canal, about $11 / 2$ inches in length, occupies the lower

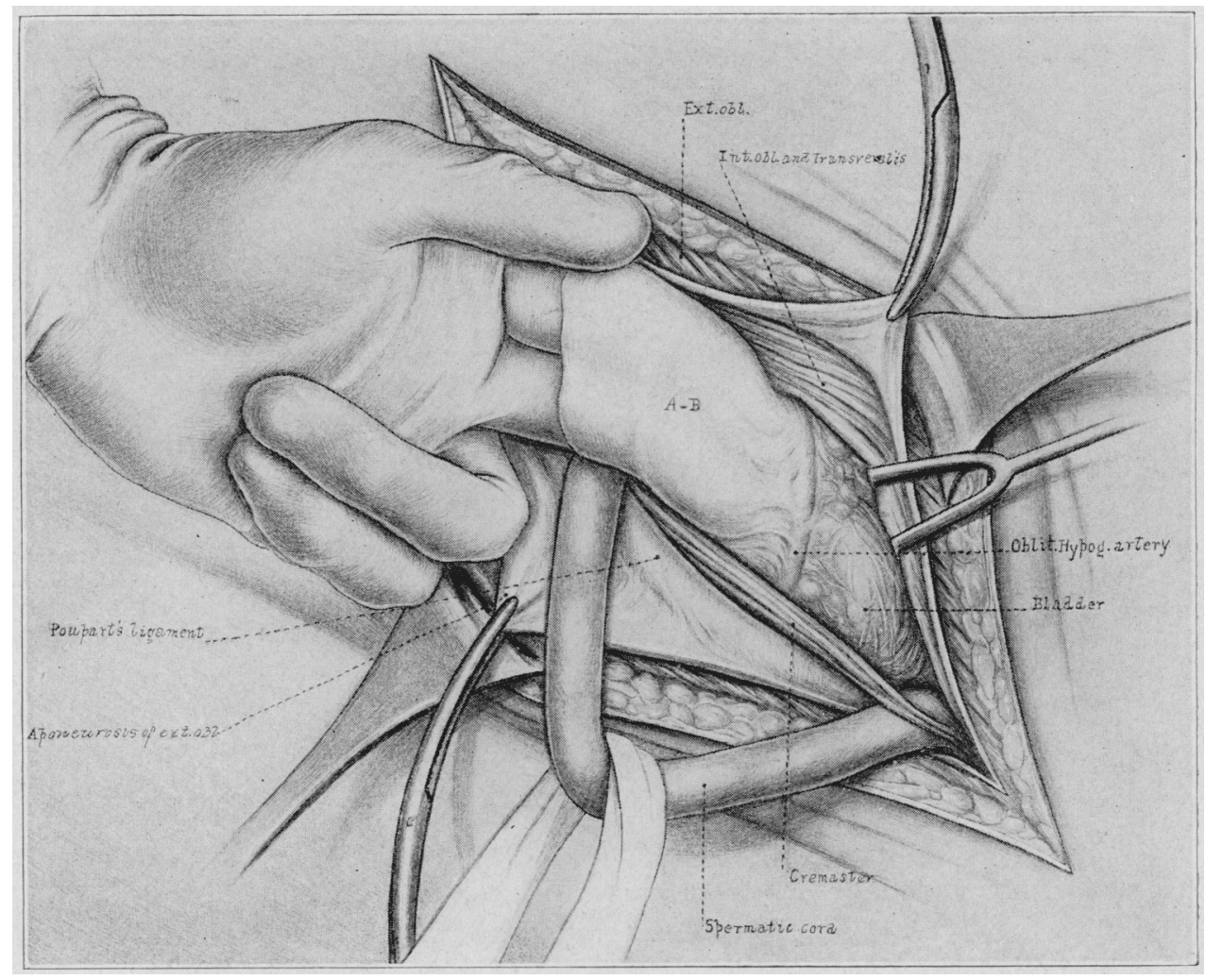

Fig. 7.- Sac opened showing properitoneal fat and bladder.

outer part of this area, and runs down and in from the internal abdominal ring to the external ring at the pubic spine. It is occupied by the cord in the male, and round ligament in the female. The sacs of all hernias situated in this region bear a definite relation to this canal. The oblique or indirect hernias enter it at the internal ring and when complete traverse its entire course. The usual form of direct hernia, forcing its way through the transversalis fascia, enters 
the canal at some point below the deep epigastric artery and appears at the external ring or not, depending on the size of the sac.

Direct hernias constitute about 10 per cent. of all primary inguinal hernias. On the other hand, considerably more than 50 per cent. of secondary or recurrent inguinal hernias are of this variety. This large proportion of direct recurrences indicates either that the usual operation for this type of hernia is inadequate or that the primary hernia existed as a combined direct and indirect hernia, and the direct por-

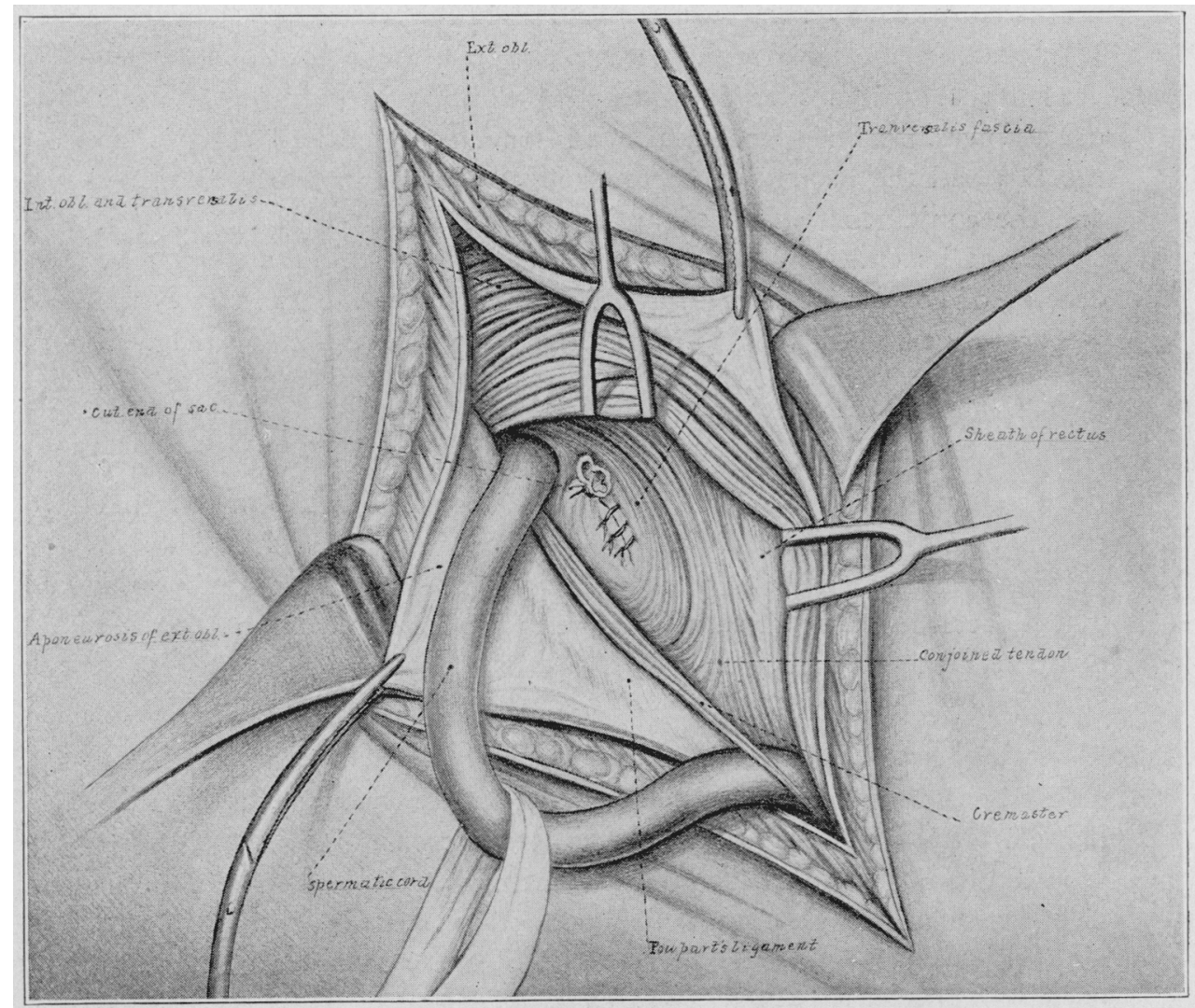

Fig. 8. -Neck of sac closed.

tion of the sac was overlooked or the cases were improperly selected. The typical form of direct hernia (Fig. 2) appears just below the deep epigastric artery through the weak middle portion of the transversalis fascia, and has, besides peritoneum, properitoneal fat and fascia, the cremaster muscle as a covering. However, not infrequently the cremaster with the cord lies entirely to the outer side of the sac. The combined direct and indirect hernia - the so-called pantaloon 
sac - one portion of which appears above the deep epigastric artery and the other below (Fig. 3), occurs perhaps half as frequently as the typical form. In this type either sac may constitute the major portion of the hernia, or the two may be of equal size. Rarely a direct protrusion occurs through the lower thick segment of the transversalis fascia some distance below the epigastric artery, and at times the sac may be divided into two portions by a band of fascia. An entirely different form of hernia, erroneously classed as direct inguinal, is occasionally found in the lower inner portion of this region (Fig. 16). It appears in the linea semilunaris just above the pubic bone, and is very probably due to properitoneal fat working its way through one of the perivascular spaces. The peritoneum is dragged along with the fat, and the protrusion occurs between the margin of the rectus and the conjoined tendon, or pushes the latter structure ahead of it as one of its coverings. This hernia, usually small, although it may become of considerable proportions, resembles to a marked degree the femoral type in that it is globular in shape, contains much peritoneal fat, and has a narrow neck with sharp margins. The location of this protrusion is the only reason that can be given for grouping it under the general heading of direct hernia, as in no way does it conform to the essential requirements necessary for such classification. Hernia of the linea semilunaris may exist coincidentally with direct or indirect hernia, and may also occcur at other points in the linea semilunaris. These protrusions are similar in nature to the epigastric hernia situated at or near the median line.

CAUSES, SYMPTOMS AND DIAGNOSIS OF DIRECT HERNIA

Direct hernia occurs most often in men between the ages of 25 and 45. It is occasionally seen in younger persons, and has even been reported in children. It is rare in women. Poorly developed or deficient musculature in the lower half of the abdominal wall may be said to be the underlying cause in practically every instance. Many of these patients have a narrow pelvis, with Poupart's ligament and the fibers of the external oblique muscle taking an almost vertical course. The portion of the internal oblique and transversalis muscles arising from Poupart's ligament, which usually consists of heavy muscular fibers extending well down over the internal ring and upper part of the inguinal canal, may be replaced by a thinned aponeurotic layer with a short muscular belly leaving the normally weak portion of the transversalis fascia with little or no support. The rectus muscle is likely to be narrow and thin. As no preformed peritoneal pouch or protrusion exists at the point in the floor of the inguinal region where 
direct hernia usually occurs, it is, strictly speaking, classed as of the acquired variety; however, the above described condition of the muscles in this region is unquestionably of congenital origin in many cases. The form of occupation of the patient does not seem to be responsible for the development of the hernia. It is conceivable that the condition will appear earlier in those doing hard labor than in those doing light work, but it is a fact that many of these hernias are seen in tailors,

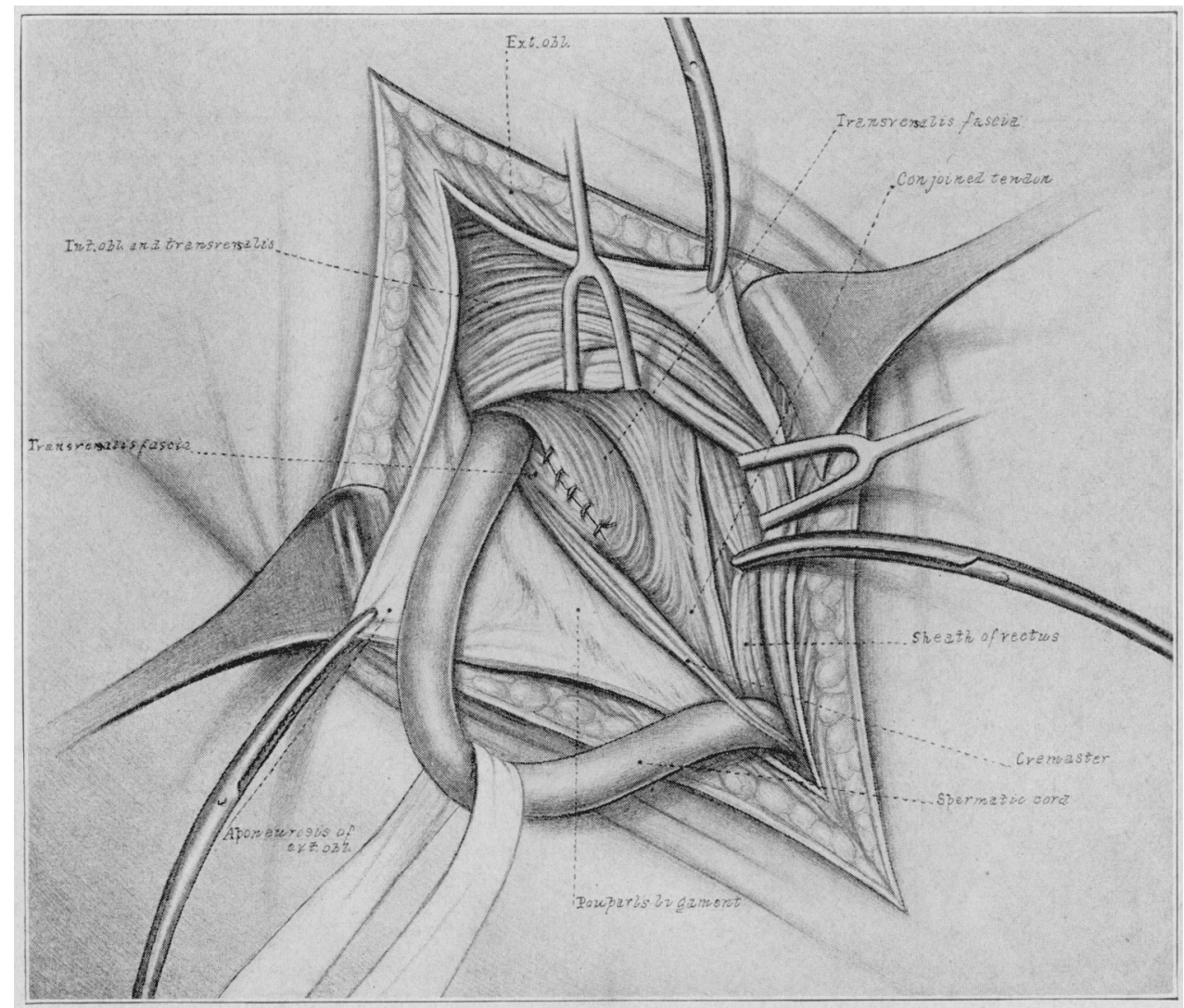

Fig. 9.-Closure of transversalis fascia as a separate layer; sheath of rectus opened.

clerks and bookeepers. The majority of direct hernias develop without giving rise to symptoms. Either the man notices a painless swelling in the inguinal region, or his attention is called to such a swelling while undergoing a physical examination for one reason or another. A certain number of patients will state that after an unusual effort at lifting or straining, they suffered severe pain in the groin and immediately noticed a swelling. Examination fails to reveal evidence 
of an acute lesion, as would surely be the case if a trauma had been received of sufficient violence to cause a palpable mass. The extra effort has suddenly forced some portion of the abdominal contents into a sac, which has slowly formed without giving rise to symptoms. Some patients complain of dull pain or a sense of weakness in the inguinal region from the incipiency of the hernia. Once the condition has developed to any extent, the symptoms are those usual to hernia in this region, with the exception that on account of the large size of the neck of the sac in the average case there is little tendency to strangulation.

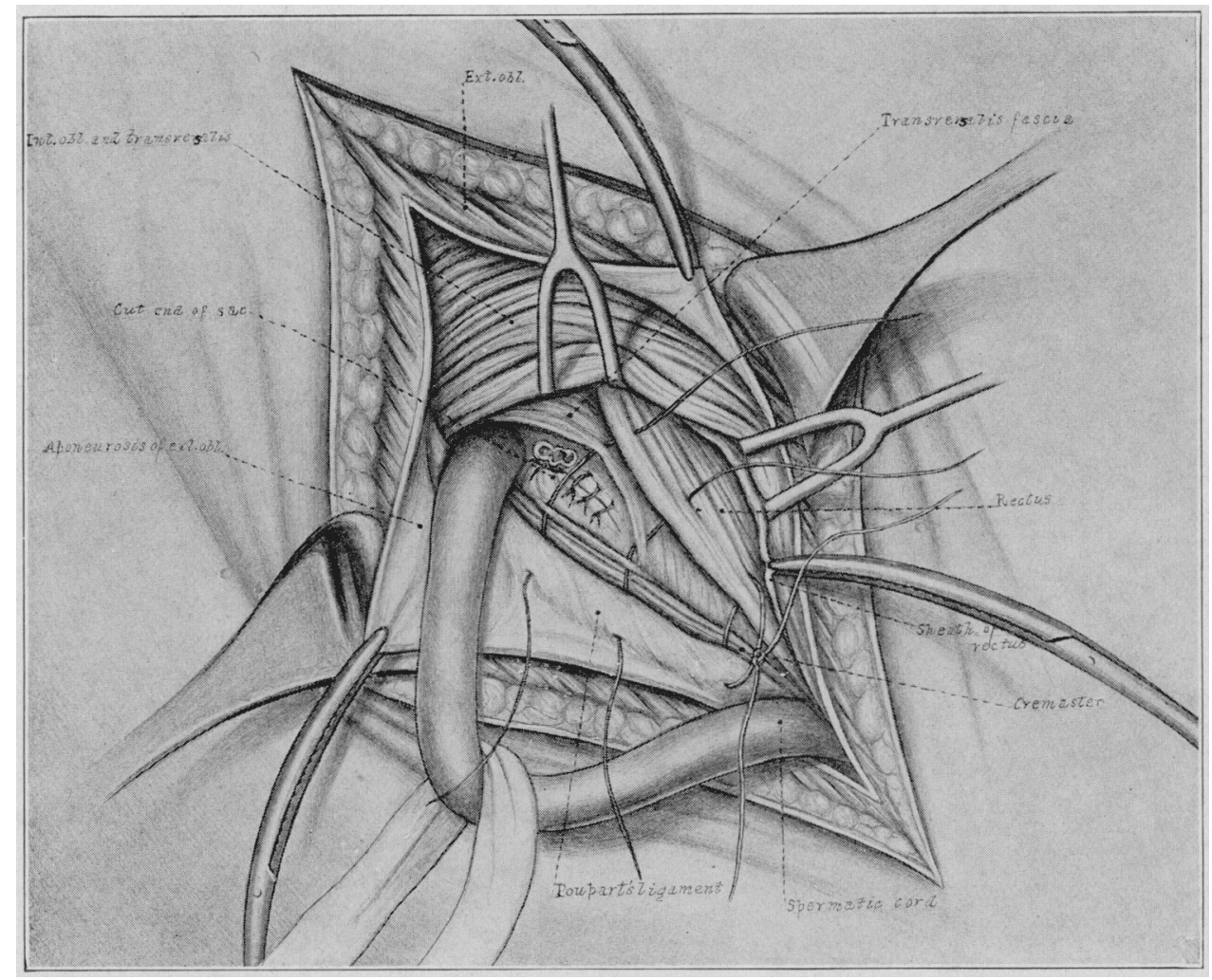

Fig. 10.-Rectus muscle exposed, sutures in place.

If these points are borne in mind, the diagnosis of direct hernia is easily made in most instances: (1) Age and sex of the patient ; (2) condition of the lower abdominal muscles; (3) the fact that the protrusion is often bilateral; (4) the situation which is low down over the pubic bone except in very early cases in which the sac has not descended to the external ring; (5) globular swelling which does not 
tend to enter the scrotum even if of large size and is easily reducible, and (6) a definite weakness disclosed by palpation over the lower portion of the inguinal canal. By invaginating the scrotum through the external ring, the finger may be passed directly back over the pubic bone, and the margin of the rectus muscle easily palpated. Occasionally, the deep epigastric artery may be felt above. On the other hand, an exceptional case of indirect hernia will present a sufficient number of the physical signs just enumerated to make the differential diag-

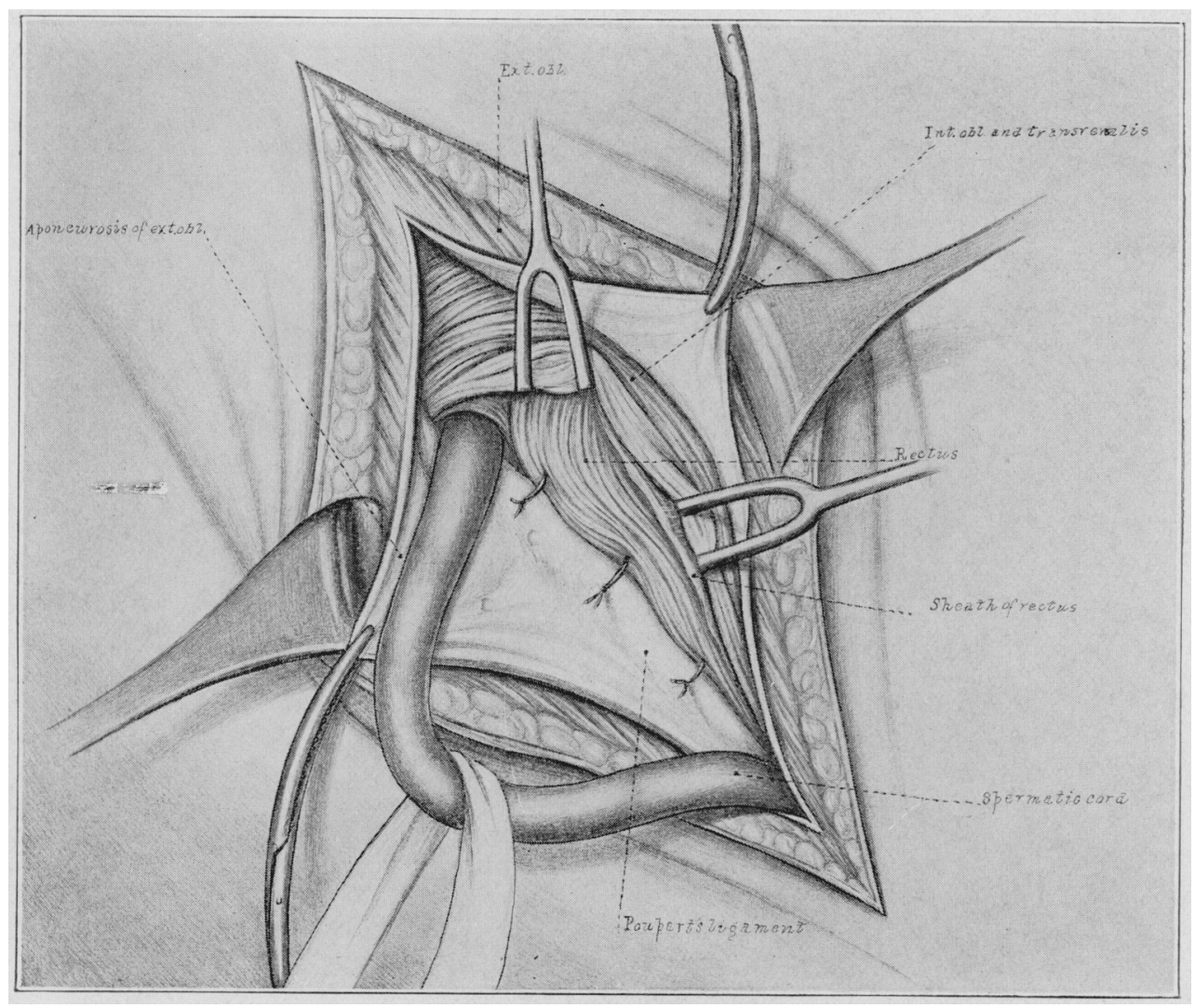

Fig. 11.-Rectus muscle in apposition to Poupart's ligament.

nosis very difficult. It is practically impossible to differentiate direct hernia from hernia of the linea semilunaris. The latter occurs usually in persons with a considerable amount of fat in the pubic region, and for this reason palpation or exploration of the canal with the finger is not satisfactory. I know of no case of hernia of the linea semilunaris in this region that was diagnosed before operation. 
TREATMENT OF DIRECT HERNIA

The results obtained in the operative treatment of direct hemina depend in a large measure on the proper selection of cases. Old men with weak muscles and relaxed tissues in general are poor subjects for the plastic type of operation necessary to cure this condition, and except for some very good reason they should not be operated on. The younger men with weak lower abdominal muscles, and an almost vertical pelvis should be examined with great care, and the effort made

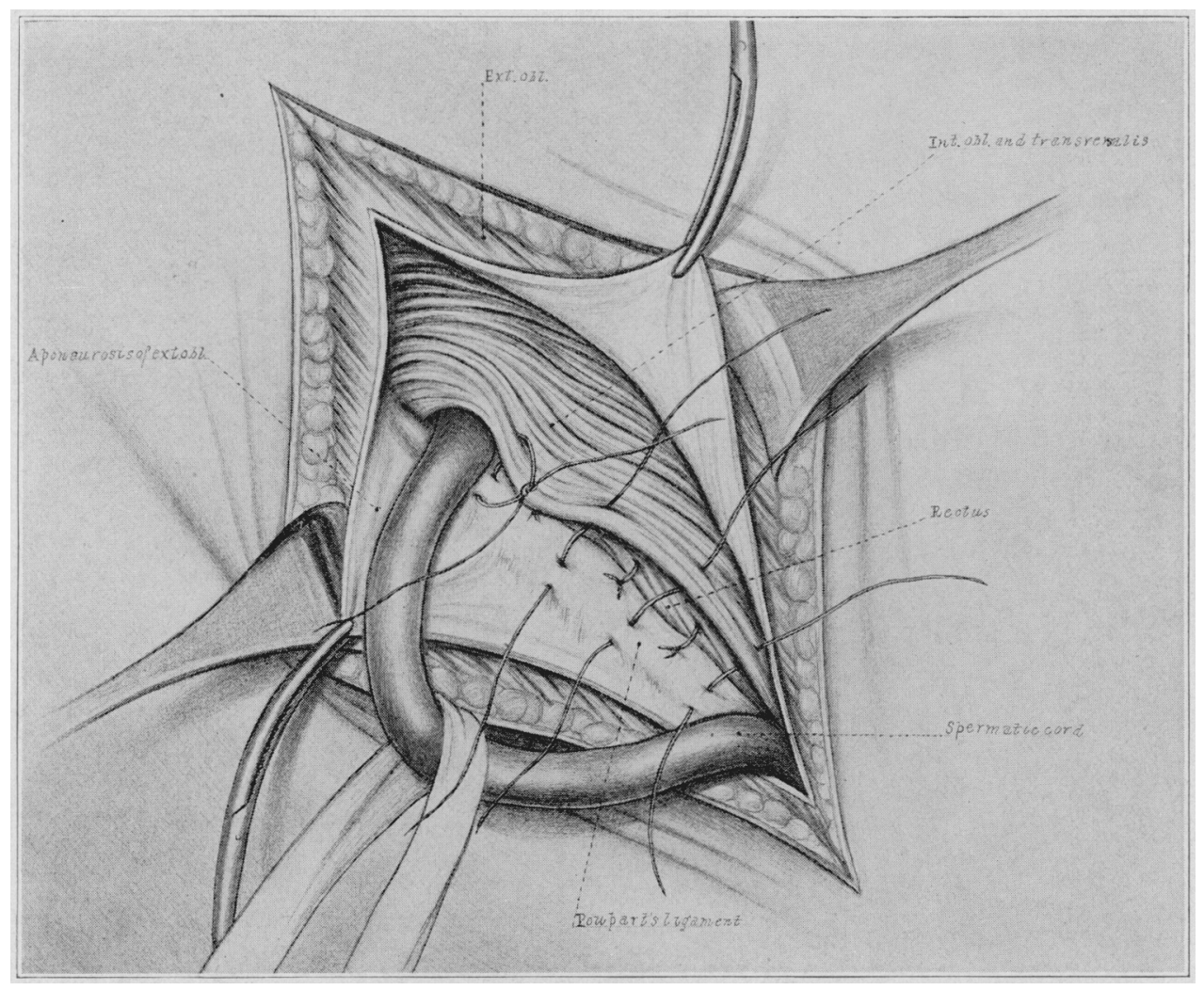

Fig. 12.-Bassini operation after rectus transplantation.

to determine whether by any sort of operation the structures in the inguinal region can be so strengthened as to resist the intra-abdominal pressure which in these patients is apparently greater than the deficient musculature can withstand. The most unfavorable cases for operation are those in which there is a protrusion usually of small size occurring about the middle of the inguinal canal in which the external oblique is relaxed and very thin, or in which there is a separation of its fibers. 
The external ring may not admit the tip of the finger; but with the patient lying down, one or more fingers may be passed through the hiatus in the external oblique muscle and the edge of the rectus muscle, or the pubic bone may be palpated. It is in this class of cases that I believe the patient's best interest will be served if a truss is applied just tight enough to balance the intra-abdominal pressure, and systematic exercise of the abdominal muscles advised. If the muscles are improved by the exercise and the hernia persists, the question of

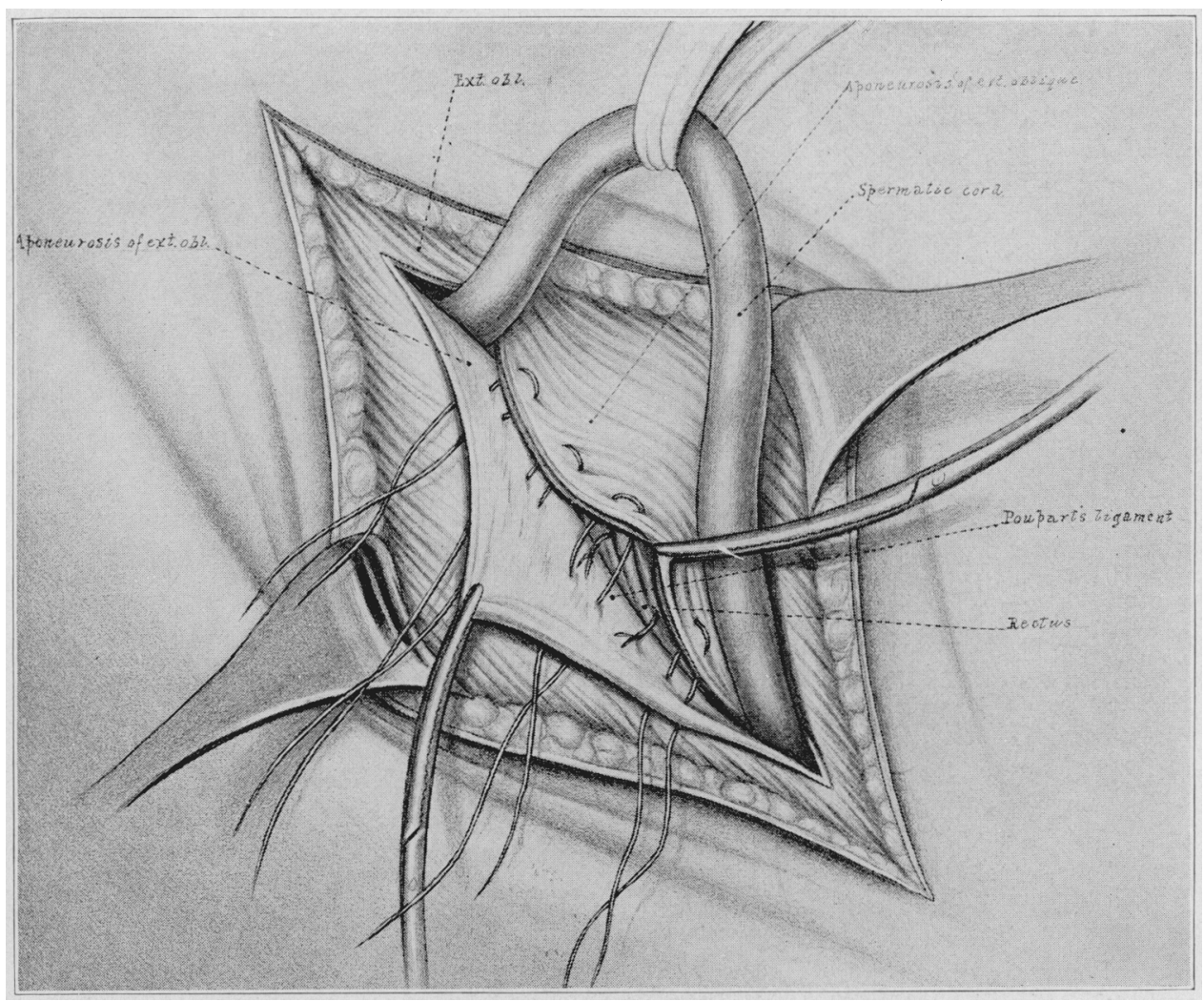

Fig. 13.-Andrews operation after rectus transplantation.

operation may again be taken up in a period of from six months to a year. No patient with a chronic cough should be operated on unless there is danger of strangulation.

In the selection of the proper operation for a given case of direct hernia, just as much care should be exercised as in the selection of cases suitable for operation. While the great majority of these hernias fall into two general groups - the usual form in which the protrusion occurs through the normal weak portion of the transversalis fascia 
and the combined direct and indirect type - yet an operation which will prove satisfactory in one case may be wholly inadequate in another. Anatomic variations explain this difference. In one instance, the muscles may be well developed, and any one of the well recognized operative procedures may give a perfect result. On the other hand, the muscles may be thinned out, poorly developed and the sac large, its base extending almost from the epigastric artery to the pubic bone. An entirely different operation is necessary if a satisfactory outcome is to be expected under such conditions. In spite of the fact that it is generally known that the structures differ in this way, many writers continue to group all direct hernias under one heading, and advise a given operative technic for all cases. Frequently, the operation recommended is one of the recognized methods used for the cure of oblique hernia. That this type of operation is inadequate in cases of direct hernia in which the muscles are undeveloped and the conjoined tendon absent or attenuated was recognized by Bloodgood ${ }^{1}$ twenty years ago. This author, in an exhaustive report of cases in which operation was performed at the Johns Hopkins Hospital, showed conclusively that if satisfactory results were to be obtained in this form of rupture, something more than the routine technic must be employed. About 1909 , I began the use of the transplanted or, more properly speaking, transposed rectus muscle in conjunction with the Bassini operation in the treatment of this class of cases. So many recurrences were taking place in my patients as well as in those of other surgeons with whom I was associated that it became quite evident that the Bassini operation alone did not meet the indications. Accordingly, I adopted the following method, which I have modified from time to time.

\section{OPERATION}

The usual skin incision is made, carrying the lower angle down to the pubic bone, as it is important to have a good exposure in the region of the external ring. The aponeurosis of the external oblique is divided well over toward the edge of the rectus muscle, and both flaps are retracted. The sharp margin of Poupart's ligament is then exposed down to the pubic spine. By blunt dissection, the fibers of the cremaster muscle are separated and the cord gently lifted from its bed. A small retractor is now placed under the arched fibers of the internal oblique and transversalis muscles, exposing the internal ring. By gentle traction on the cord, the peritoneal reflection at the internal ring is brought into view and the presence or absence of an oblique hernia quickly determined. This step is absolutely essential and should be carried out before attempting to isolate the direct hernial sac irrespective of its size or location, for in no other way can the error of overlooking the indirect portion of a combined sac be avoided. If no indirect sac is found, the direct is opened on its upper side in order to keep as far away from the bladder as possible. I believe that the sac should be opened in every hernia that is of sufficient size to warrant operation.

1. Bloodgood, J. C.: Johns Hopkins Hospital Report, 1899. 
In the well developed cases, the transversalis fascia is thinned out, and as a rule there is little properitoneal fat. In the smaller ones the fascia may be quite firm and a large amount of fat present, causing considerable difficulty in locating the sac. The outer surface of the sac on its lower and inner sides is more or less covered by fat - usually spoken of as bladder fat - but which in reality differs in no wise from the properitoneal fat of other locations. With the finger inserted through the neck of the sac for support, the fat is gently stripped from its surface. As its lower limits is approached, a number of small blood vessels will be seen and a small tonguelike projection of bladder

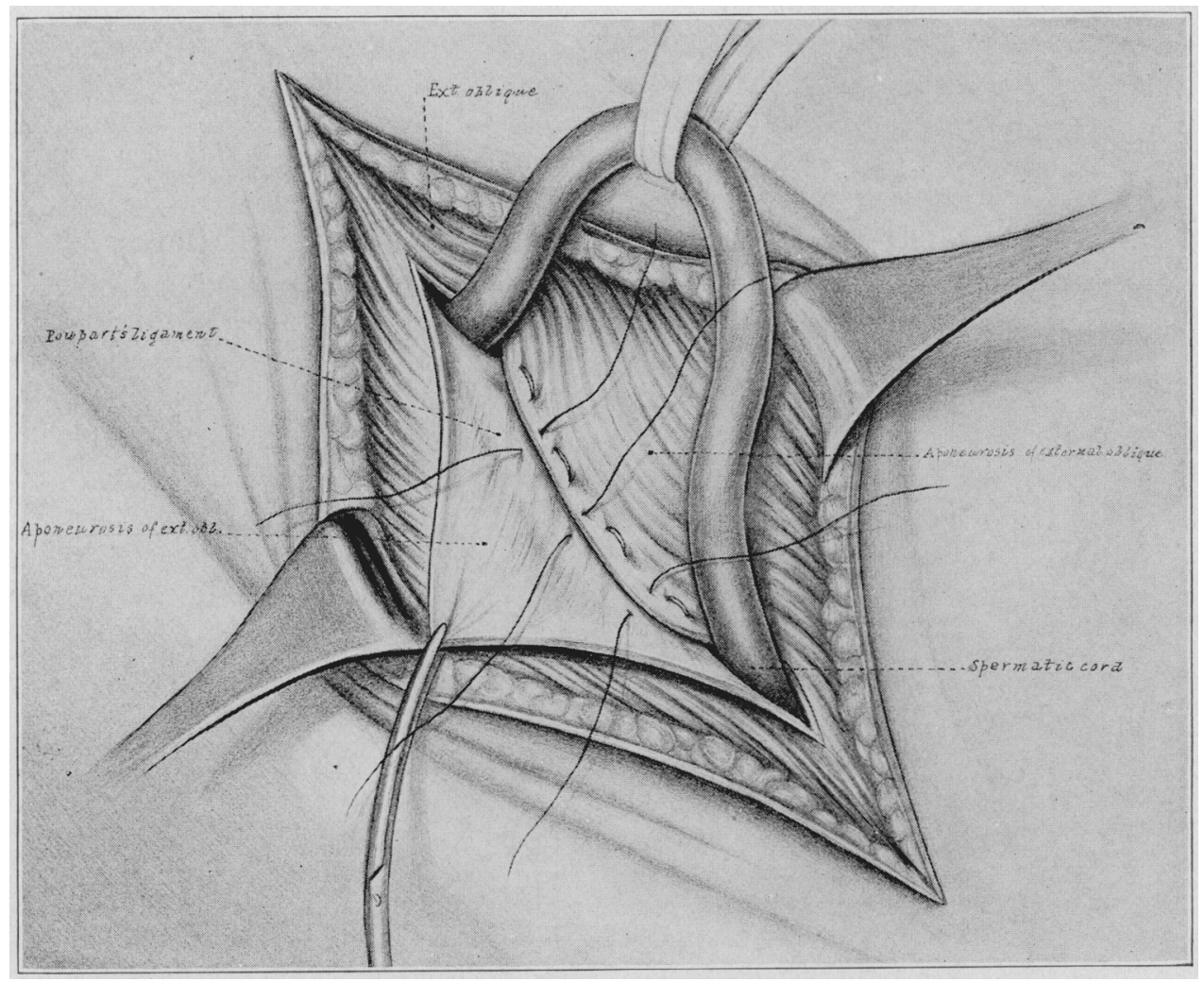

Fig. 14.-Suture of external oblique to Poupart's ligament. Continuous suture is preferred to interrupted, as illustrated.

muscle observed in most cases (Fig. 7 ). These muscle fibers are rather pale in color and run somewhat parallel to the long axis of the sac. With ordinary care, the bladder should never be injured. The fat is stripped readily as a rule, but occasionally it may be found adherent from the long continued use of a truss. Frequently the obliterated hypogastric artery, a dense fibrous band, will be encountered as the stripping proceeds toward the bladder. This structure, often referred to as indicating the lowest limit to which the fat may safely be removed, is of no value whatsoever as a guide to the bladder. Its location varies in nearly every case. At times it is taut, dividing the fatty mass into 
or below it at will. It should be cut if it interferes with proper dissection. After the sac wall has been satisfactorily exposed, its base should be drawn flush with the opening in the fascia and transfixed; or, better still, if of large size, sutured as in the manner of closing the peritoneum in laparatomy wounds (Fig. 8). I always use No. 1 chromicized catgut for this purpose.

In the cases of combined direct and indirect hernia, the two portions of the sac should be converted into one, by drawing one or the other above or below the deep epigastric artery, being guided by their relative size as to whether it is best to convert the direct into an indirect or vice versa (Figs. 4 and 5). I formerly advised dividing the artery in this type of hernia (Fig. 6), but I am

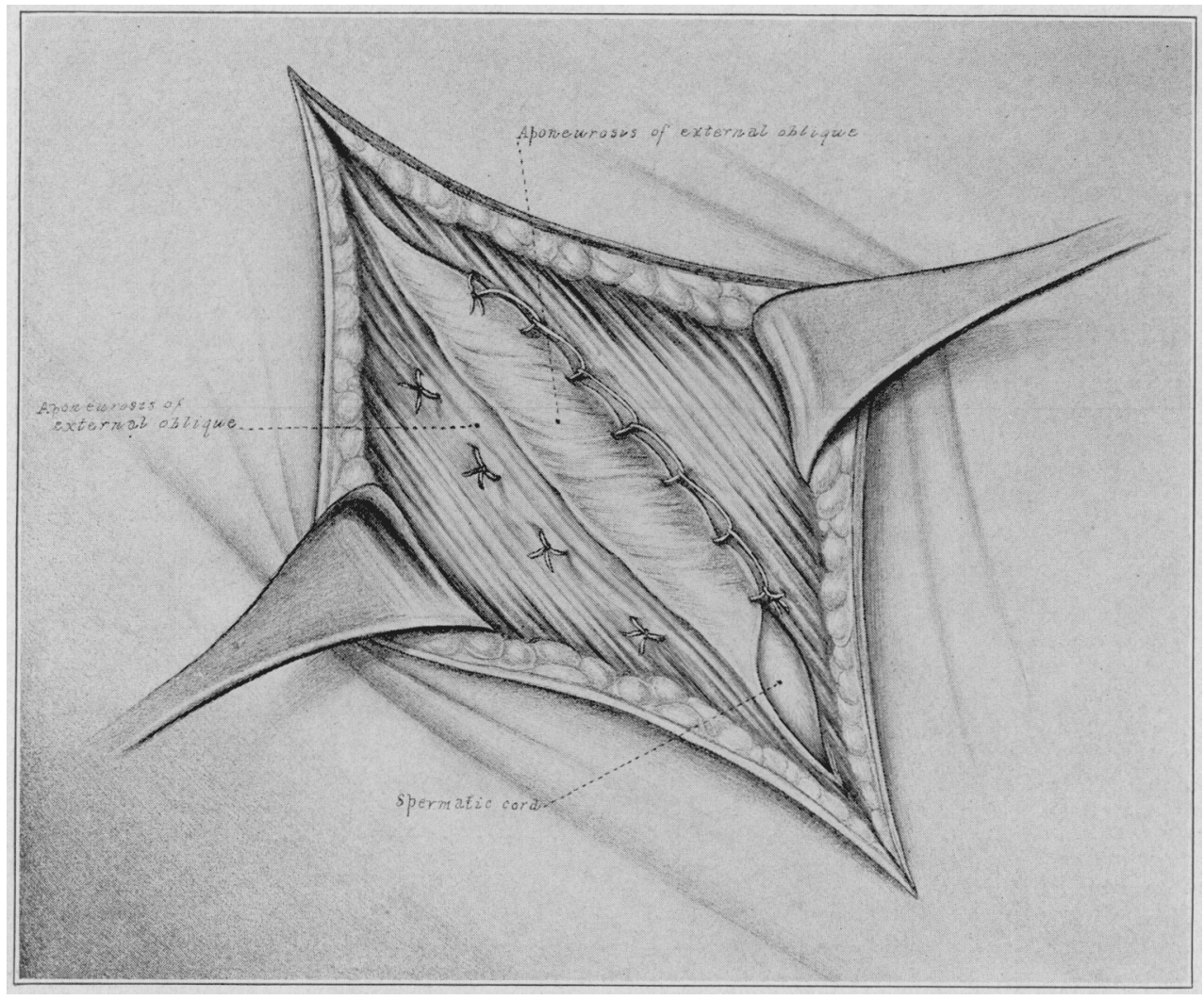

Fig. 15.-Andrews operation completed.

now of the opinion that the sac can be removed in most instances just as thoroughly without sacrificing this vessel. These hernias should all be classed as direct and handled as such. After the sac has been disposed of, the rent in the transversalis fascia should be closed if possible, a continuous suture of chromic gut being utilized for the purpose (Fig. 9). A second retractor is now placed low down under the continuation of the internal oblique and transversalis muscles, and the sharp margin of the rectus muscle located with the finger. The sheath of the rectus is then opened along its anterior border, and the muscle exposed from about the level of the internal ring down to its pubic 
attachment (Fig. 9). Three to four sutures of kangaroo tendon are introduced between the outer portion of the rectus muscle and the deepest part of Poupart's ligament. If the transversalis fascia has not been closed satisfactorily as a separate layer, it should be included with these structures. The sutures should be placed from one half to three quarters inch apart and inserted from below up - the lowest one passing from just above the insertion of the rectus muscle to the terminal portion of the ligament (Fig. 10). After they have been properly placed, gentle traction should be made on the sutures drawing muscle and ligament well together, and while thus held by an assistant, they should be tied in the order of their insertion (Fig. 11). The usual Bassini operation

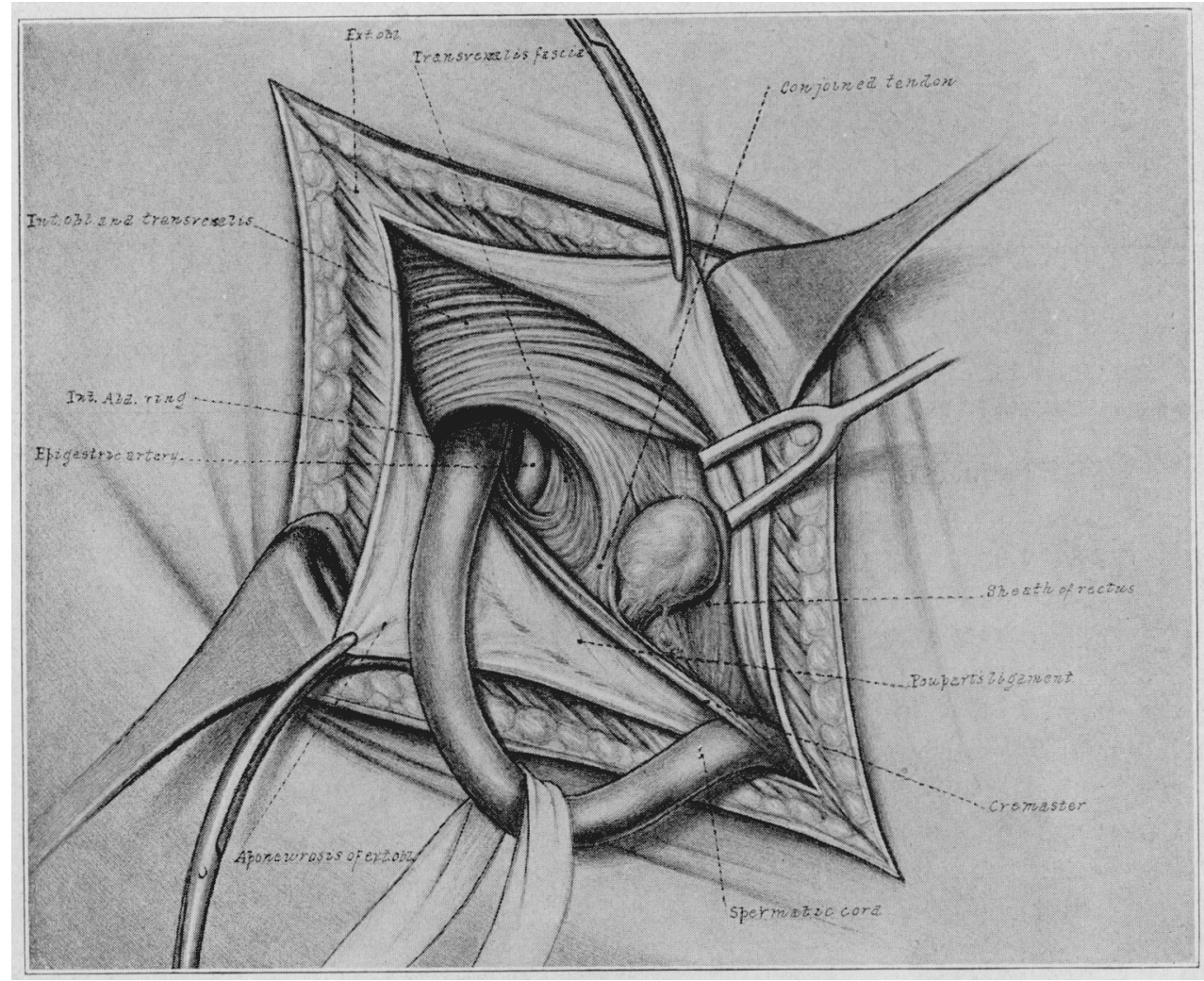

Fig. 16.-Hernia of linea semiluraris.

is now performed from above downward, the sutures picking up a small bite of the rectus muscle and catching Poupart's ligament just superficial to and between those of the first row (Fig. 12). Excessive fat should be removed from the cord, but the veins should not be excised. In closing the external oblique, care should be taken to restore the external ring to as near normal as possible. The hernia of the linea semilunaris in this region is closed by removal of the sac, and the use of one or two mattress sutures placed between the margin of the rectus sheath and the conjoined tendon and transversalis fascia followed by the Bassini or Andrews operation. As these hernias are in close proximity to the bladder, the sac should be dissected with great care. 
COMMENT

Complete removal of the sac is the most important step in the operative treatment of any form of hernia, but it is necessary to provide sufficient support at the weakened area in the abdominal wall in order to prevent recurrence. In indirect inguinal hernia, proper use of the internal oblique and transversalis muscles satisfactorily overcomes this weakness; but in the direct type, these muscles alone do not meet the requirements. By making use of the rectus muscle, an additional layer is added to the weakened posterior wall of the canal. It is not asserted that the fibers of this muscle form a true union with Poupart's ligament or that they always remain permanently in the new position, but they do aid greatly in the formation of new connective tissue at a point where every bit of additional support is of value in the prevention of recurrence. By the use of the foregoing method, recurrences in my cases have been reduced more than half, and now that I am selecting the cases for operation with greater care, I believe that the permanent cures will show a still further increase.

Eucouraging, however, as these results may be in some ways, I feel that there is still room for improvement in the operative treatment of direct hernia. With this object in view, my assistant, Dr. R. W. Bolling, and I have recently decided to combine transplantation of the rectus muscle with the Andrews operation instead of the Bassini in the hope that this combination will give even better results (Fig. 13). The mattress sutures are placed as recommended by Andrews for his posterior operation with the addition of a continuous suture between the margin of the external oblique flap and Poupart's ligament (Fig. 14). This stitch of fine gut insures closer contact between these aponeurotic structures. The lower flap of the external oblique muscle is then sutured over the cord in the usual way (Fig. 15). If there seems to be tension in the upper flap of the external oblique muscle, a free liberating incision may be made through the sheath of the rectus well back over the belly of the muscle and parallel with its fibers. It might be said in passing that sutures placed under tension are of little or no value as they soon cut out. Kangaroo tendon or chromic catgut was used for all deep sutures up to the time of changing methods, but we are now trying celluloid linen in one series and absorbable sutures in another.

\section{COMPLICATIONS}

Two accidents occurred in this series of cases - both about ten years ago. One was in a recurrent hernia in which the vas was cut as the incision was made through the skin. The cord had been placed superficial to the external oblique aponeurosis at the original operation. In the other, a large hernia with much properitoneal fat, the 
bladder was opened, but the injury was recognized and no ill effect followed. This is the only time I have ever seen the bladder opened during an operation for hernia, nor have I ever seen the iliac artery or vein injured in this operation. Hematoma of the cord seldom follows operations for direct hernia, owing to the fact that the sac is rarely adherent to this structure. Postoperative hydrocele is of such rare occurrence after operation for this form of hernia that I would almost be inclined to look on this condition as a coincidence rather than a result. As a matter of fact, the various forms of tumefaction in the scrotum referred to by Seward Erdman ${ }^{2}$ as occurring after the hernia operation are rarely seen, provided the cord is managed correctly during the operation, and the scrotum properly supported by a well fitting suspensory afterward. In no instance has atrophy of the testicle been noted in following up these cases. The patient in which the vas was divided was lost track of shortly after operation.

Since 1909, I have operated by the method referred to, that is, rectus transplantation combined with the Bassini operation, on 249 patients with direct hernia. In sixty-nine, the hernia was bilateral, making a total of 318 operations. Ninety patients have been followed for one year or longer, including twenty-three in whom the hernia was double. In this total of 113 operations, there have been six recurrences, or a recurrence of somewhat less than 5.5 per cent. It is suggestive that three of the recurrences followed operation for bilateral hernia performed at one operation.

\section{CONCLUSIONS}

1. The patient with a direct inguinal hernia should be carefully studied before recommending operation. If the condition of the lower abdominal muscles is such that a fair chance of obtaining a cure cannot be offered, the question of operation should be deferred for the time being and the patient advised to take systematic exercises for a period of from six months to one year.

2. There is a small but definite group of patients with poorly developed muscles on whom it is not wise to operate for this condition, as recurrence is almost certain to follow.

3. Complete removal of the sac and the use of the rectus muscle in conjunction with one of the recognized operations for indirect inguinal hernia have reduced our percentage of failures in direct hernia more than half.

2. Erdman, Seward: Ann. Surg. 66:702 (Dec.) 1917. 